\title{
Sobre la Enseñanza de la Distribución de Poisson en Carreras de Ingeniería*
}

\section{On the Teaching of Poisson Distribution in Engineering Education}

\author{
Miguel Cruz-Ramírez ${ }^{* *}$ \\ Salvador Eladio Álvarez-Reyes ${ }^{* * *}$ \\ Francisco Javier Pérez-Santos ${ }^{* * * *}$
}

\begin{abstract}
Resumen
En el presente estudio se examinan dos metodologías para la enseñanza de la distribución de Poisson en carreras de ingeniería. La primera se basa en la problematización del contenido, considerando la distribución de Poisson como aproximación a la distribución Binomial. La segunda metodología se apoya en las relaciones dialécticas de inducción-deducción, siguiendo el enfoque de un proceso de Poisson. Cada vía se implementa sendos grupos de estudiantes de ingeniería, con el objetivo de indagar sobre el efecto que ocasionan en el aprendizaje de las distribuciones. El aprendizaje se concibe de manera desarrolladora, en el sentido de integrar los procesos de interiorización y exteriorización del conocimiento. En ambos grupos experimentales, los resultados resultan superiores una vez finalizado el estudio, pero solo en el segundo se aprecian efectos estadísticamente significativos. Las evidencias empíricas no revelan superioridad de una metodología respecto a la otra, lo cual pone de manifiesto que el desarrollo lógico-matemático es menos determinante en lo gnoseológico que el método establecido desde una perspectiva didáctica.
\end{abstract}

Palabras-clave: Distribución de Poisson. Probabilidades y Estadística. Formación de Ingenieros.

\begin{abstract}
In the present study, two methodologies related to the teaching of Poisson's distribution were examined in the field of engineering education. The first methodology is based on a problematic way around the content domain, considering this distribution as approximation to Binomial distribution. The second methodology, based on the dialectic relations of induction-deduction, goes along a focus of Poisson's process. Both ways apply in two engineering careers, for the sake of evaluating the effect that they cause in learning. The learning is focused as developer, in the sense of integrating the processes of internalization and exteriorization of knowledge. The learning of probability distributions gets better in both experimental groups, but only upon the second way it is appreciated a statistically significant effects. The empiric evidences do not reveal superiority of a methodology in relation to the other. From a gnoseological perspective, it showed that the logical and mathematical development is less determining than the method established from the didactic point of view.
\end{abstract}

\footnotetext{
* Investigación financiada por el proyecto AECID A2/039476/11 de la Universidad Politécnica de Valencia: Fortalecimiento Institucional en la Enseñanza de la Estadística, la Investigación Operativa, Fiabilidad, y Calidad y Aplicación Científica en Temas de Interés Regional.

** Doctorado en Ciencias Pedagógicas de la Universidad de Ciencias Pedagógicas José de la Luz y Caballero (UCPJLC). Profesor Titular de la Universidad de Holguín Oscar Lucero Moya (UHOLM), Holguín, Cuba. Dirección postal: Carretera vía Guardalavaca, Rpto. Piedra Blanca, Holguín 81000, Cuba. E-mail: mcruzr@facinf.uho.edu.cu, cruzramirezmiguel@gmail.com.

**** Doctorado en Ciencias Pedagógicas de la Universidad de Erfurt (UE). Profesor Auxiliar de la Universidad de Holguín Oscar Lucero Moya (UHOLM), Holguín, Cuba. Dirección postal: Carretera vía Guardalavaca, Rpto. Piedra Blanca, Holguín 81000, Cuba. E-mail: sar@facinf.uho.edu.cu.

${ }_{* * * * *}^{*}$ Licenciado en Ciencias Económicas de la Universidad de Holguín Oscar Lucero Moya (UHOLM). Profesor Auxiliar, Universidad de Holguín Oscar Lucero Moya (UHOLM), Holguín, Cuba. Dirección postal: Carretera vía Guardalavaca, Rpto. Piedra Blanca, Holguín 81000, Cuba. E-mail: fperez@ facinf.uho.edu.cu. 
Keywords: Poisson Distribution. Probability and Statistics. Engineering Education.

\section{Introducción}

Cada día se amplía más el espectro de trabajo de los ingenieros. El desarrollo de nuevos productos, el diseño de prototipos, el perfeccionamiento de herramientas, máquinas y procesos, el mantenimiento, la localización y reparación de averías, son ejemplos bien simplificados del amplio quehacer ingenieril. En cada una de estas funciones los ingenieros coleccionan y analizan datos. Al respecto Bisgaard (1991, p.274) ha señalado que: “[...] hayan aprendido o no estadística, ellos harán estadística. Por tanto, el asunto no es si utilizan estadística o no, sino cuán bien deben hacerlo". Por tal motivo, el aprendizaje de contenidos estadísticos constituye un aspecto importante en la formación universitaria de estos profesionales (HECKEL, 1994; HOGG, 1994; BENDELL; DISNEY; MCCOLLIN, 1999; BLANCO, 2008).

Por un lado, estudios curriculares abordan extensamente la formación inicial y permanente de ingenieros mientras que, por otro, los estudios didácticos tratan aspectos cada vez más complejos de la enseñanza y el aprendizaje de la estadística. La intersección de estos dos campos constituye, sin embargo, un tema poco explorado (SORGE; SCHAU, 2002). En general, se aboga por el empleo de métodos activos que estimulen el aprendizaje cooperado (KVAM, 2000), por la necesidad de centrarse más en el estudiante que en el profesor (ROMERO et al., 1995), por el estudio del significado que adquieren los conceptos estadísticos (OLIVO, 2008), incluso por la urgencia de no desatender la relación entre los métodos cualitativos y cuantitativos en la práctica profesional de los ingenieros prospectivos (BORREGO; DOUGLAS; AMELINK, 2009), entre varios aspectos.

Tradicionalmente, los cursos del nivel terciario tratan sobre el uso de procedimientos para el procesamiento de datos, teoría de probabilidades, distribuciones univariada y bivariada, estimaciones de parámetros, prueba de hipótesis y análisis de regresión. Al respecto, Zewotir y North han criticado la tendencia a hacer más hincapié en la teoría y menos en las aplicaciones de la estadística. Al fomentar un enfoque dirigido hacia adentro, donde la teoría juega un rol dominante, seguida de unas pocas técnicas, desacierta el supuesto de que la valía del propio tema hablará por sí misma (ZEWOTIR; NORTH, 2007).

A menudo se observa una ruptura temporal entre el aprendizaje de la estadística en los años iniciales y su puesta en práctica en los años terminales. Incluso se ha reportado que, a la altura de la culminación de estudios, los estudiantes de ingeniería vienen a comprender la 
importancia de la estadística en su formación profesional (JOHNSON et al., 2008), pero la perdurabilidad y solidez del conocimiento resulta insuficiente. Por esta razón, también las investigaciones abogan por elevar la calidad de la enseñanza de la estadística desde los cursos introductorios (ACOSTA, 2000).

Un concepto de vital importancia en el aprendizaje de los contenidos estadísticos es el concepto de distribución, el cual está asociado a la idea primaria de variabilidad (WILD, 2005). Recientemente se han examinado relaciones cognitivas profundas entre variabilidad y distribución (WATSON, 2009). Particularmente, la aprehensión del concepto de distribución muestral es crucial para la comprensión de las ideas de intervalo de confianza y nivel de significación (DYCK; GEE, 1998; LIPSON, 2003).

Tal y como ocurre con los conceptos matemáticos en sentido general, los problemas de aprendizaje asociados a las distribuciones suelen ser diversos, e incluso tienden a persistir después de la instrumentación de métodos activos y nuevas tecnologías (SALDANHA; THOMPSON, 2001). No obstante, algunos autores han reportado que existen evidencias sobre la posibilidad de investigar el desarrollo de las concepciones de los estudiantes sobre los conceptos de variabilidad y distribución, sin la necesidad de efectuar cambios en el currículo (READING; REID, 2006).

Frecuentemente, los estudios sobre el aprendizaje de las distribuciones se enfocan hacia cuestiones generales que, si bien son necesarias, pasan por alto las especificidades de las propias distribuciones. En el presente trabajo se enmarca en la enseñanza de la distribución de Poisson, la cual es muy importante por la amplia variedad de problemas prácticos a los cuales sirve de modelo matemático.

\section{En busca de un aprendizaje desarrollador de la distribución de Poisson}

Son poco frecuentes los estudios sobre problemas didácticos asociados al aprendizaje de distribuciones específicas. Sin embargo, problemas relacionados con la distribución de Poisson suelen emplearse con propósitos afines al aprendizaje de la teoría de probabilidades, de manera general (CATRAMBONE; HOLYOAK, 1990).

La mencionada distribución debe su nombre a Siméon Denis Poisson (1781-1840), probabilista francés quien fue el primero en describirla en 1837. Se trata de una distribución discreta de probabilidad muy útil, en la cual la variable aleatoria representa el número de eventos independientes que ocurren a una velocidad constante. Considerando un intervalo de tiempo determinado, sirven de ejemplos el número de personas que llegan a un mercado, el 
número de llamadas telefónicas que se reciben en una central de teléfonos, el número de defectos en piezas similares en un proceso productivo, el número de bacterias presentes en un cultivo, entre otros.

En el orden matemático, la distribución de Poisson suele desarrollarse desde dos perspectivas diferentes: como aproximación a la distribución binomial $B(n, p)$, o como un proceso de Poisson. Desde la categoría didáctica medio de enseñanza, es factible la coexistencia de ambos derroteros, pues los autores de libros de texto siguen, indistintamente, uno u otro camino al introducir el nuevo contenido (FREUND; MILLER; JOHNSON, 2006). Sin embargo, el asunto no está del todo claro desde la categoría método de enseñanza.

Es deseable la consecución de resultados positivos en el proceso de enseñanzaaprendizaje de la distribución de Poisson, en el sentido de la solidez y perdurabilidad del conocimiento adquirido, de la aplicabilidad en el marco del perfil profesional y de la importancia de este contenido en diversos campos de la ingeniería. No obstante, numerosos investigadores han señalado que subsisten dificultades en el aprendizaje de las distribuciones estadísticas, donde la distribución de Poisson constituye un caso particular (DYCK; GEE, 1998; SALDANHA; THOMPSON, 2001; LIPSON, 2003; WILD, 2005; READING; REID, 2006; WATSON, 2009).

A tenor de la existencia de dos desarrollos matemáticos para esta distribución, es atinado examinar el efecto de escoger uno u otro desde una perspectiva didáctica. Es decir, cómo incide lo lógico en lo gnoseológico en el marco de un ambiente de aprendizaje para la distribución de Poisson. Esto no significa que se deje de mostrar ambos caminos, sino de indagar cuál de ellos resulta más viable en el proceso de aprehensión de los nuevos conocimientos. Tal viabilidad se explica seguidamente, en un sentido desarrollador.

Estudios recientes han concedido especial importancia al rol que juega el ambiente de aprendizaje en la formación de ingenieros (MAAT; ZAKARIA, 2010), lo cual puede incidir favorablemente o no. Algunas investigaciones asumen, como base epistémica, una concepción desarrolladora del proceso de enseñanza-aprendizaje, significando un enfoque personológico, consciente, transformador y cooperativo. De acuerdo con Castellanos et al.:

[...] el aprendizaje desarrollador es aquel que garantiza en el individuo la apropiación activa y creadora de la cultura, propiciando el desarrollo de su autoperfeccionamiento constante, de su autonomía y autodeterminación, en íntima conexión con los necesarios procesos de socialización, compromiso y responsabilidad social (CASTELLANOS et al., 2001, p. 33).

Desde esta perspectiva, el rol fundamental del docente es actuar como mediador en el proceso de desarrollo del alumno. Así, en un sentido vigotskiano, el énfasis en las 
potencialidades garantizará el tránsito por estadios de desarrollo cada vez superiores. Varios autores han destacado la necesidad de crear una atmósfera de confianza, seguridad y empatía en la clase, donde se favorezca el análisis y se respeten los juicios en un ambiente participativo y creador. Para ello se requiere un correcto seguimiento al diagnóstico y que, en base a las dificultades identificadas, se proyecten estrategias diferenciadas y compensadoras, apoyadas tanto en el aprendizaje individual como en el aprendizaje cooperado.

\section{Explorando dos caminos para la enseñanza de la distribución de Poisson}

La presente investigación comienza con una reunión metodológica ${ }^{1}$ donde asiste el colectivo de docentes de estadística e investigación operativa del Departamento de Matemática de la Universidad de Holguín “Oscar Lucero Moya” (UHOLM). La conducción de la reunión corre a cargo del jefe de la disciplina y el objetivo fundamental consiste en establecer procedimientos efectivos para el aprendizaje desarrollador de la distribución de Poisson. Tras el debate se llega a consenso en que ambas formas pueden ser efectivas, pero que cada una requiere de un enfoque didáctico específico. Es asentido el criterio de que esta apreciación, aunque se intuye de la experiencia profesional, es subjetiva y requiere de mayor argumentación teórica y contrastación empírica.

A continuación, se conforman dos subgrupos para el análisis didáctico de sendos caminos conducentes al mismo concepto. Cada subgrupo identifica fortalezas y debilidades y, en base a esto, propone una metodología para la enseñanza de la distribución de Poisson según la vía correspondiente. Seguidamente, se produce un análisis colectivo de las dos metodologías, a fin de examinar la viabilidad y ajuste al desarrollo cognitivo de los estudiantes de las carreras de ingeniería. A continuación se detalla cada una de las metodologías.

\subsection{La distribución de Poisson como aproximación a la distribución binomial}

Para el tratamiento de la distribución de Poisson, como aproximación de la binomial, se consideraran cuatro etapas: (1) Reactivación de los conocimientos previos, (2) Creación de situaciones problemáticas, (3) Presentación del nuevo contenido, e (4) Identificación del concepto en ejemplos de aplicación.

\footnotetext{
${ }^{1}$ En Cuba se utiliza la terminología reunión metodológica para referir una reunión de análisis y discusión de problemas didácticos, cuyo objetivo fundamental es la toma de decisiones para resolver problemas de enseñanza y aprendizaje (MES, 2007, Artículo 50). 
Un factor fundamental que influye en el aprendizaje se refiere a la relación existente entre el nuevo conocimiento que el estudiante debe adquirir y sus conocimientos previos (etapa 1). Por ello, a la hora de abordar la distribución de Poisson, es conveniente puntualizar aspectos fundamentales relacionados con la distribución binomial. El recordatorio de los conocimientos previos puede realizarse a partir de problemas, en cuya solución participen activamente los estudiantes. Con ese fin, puede orientarse el análisis de situaciones en las cuales el sujeto se enfrente ante un nuevo conocimiento sin disponer de toda la información necesaria. Son estas las situaciones que Borasi (1986) denomina problemáticas, pues su formulación suele ser relativamente vaga y propicia el establecimiento de nuevas conjeturas; los métodos de aproximación pueden ser diversos y la exploración del contexto, así como las sucesivas formulaciones del problema, son fundamentales. Como ejemplos pueden servir las siguientes situaciones problemáticas:

Situación 1. En un proceso fabril de artículos de vidrio ocurren defectos por la presencia de burbujas, lo cual deja la pieza indeseable para su comercialización. Como promedio, uno de cada 10 de estos artículos tiene una o más burbujas. Determinar la probabilidad de que: (a) una muestra aleatoria de 10 tenga 3 artículos con burbujas; (b) una muestra aleatoria de 15 tenga al menos 4 artículos con burbujas.

Situación 2. Resolver el problema anterior considerando que, como promedio, uno de cada 1000 de estos artículos tiene una o más burbujas. ¿Cuál es la probabilidad de que una muestra aleatoria de 4000 tenga menos de siete artículos con burbujas?

Durante el análisis progresivo de estas situaciones, es necesario resaltar aspectos tales como:

- La identificación de la variable de interés y su clasificación. Aquí la variable consiste en el número de artículos con burbujas que se manufacturan en el proceso de fabricación, la cual es una variable aleatoria discreta.

- La determinación de los parámetros de la distribución. Respectivamente se observa que: $n=10$ у $p=0.4 ; n=15$ у $p=0.4 ; n=4000$ у $p=0.001$.

- La constatación de propiedades que caracterizan aquellos experimentos relacionados con variables aleatorias que tienen distribución binomial. Los productos de vidrio se clasifican en dos tipos (de acuerdo a la presencia o no de burbujas); las producciones de las piezas son independientes unas de otras; y la probabilidad de éxito (que la pieza contenga burbujas) se mantiene constante de una prueba a otra, igual a 0.4 para la primera situación e igual a 0.001 para la segunda situación. 
- El hecho de que los conocimientos previos no son efectivos para dar respuesta a la segunda situación. Ello se debe a que el uso de la función de distribución binomial

$$
p(x)=\left(\begin{array}{l}
n \\
x
\end{array}\right) p^{x} q^{n-x}, \operatorname{con} \mathrm{x}=0,1,2, \ldots, \mathrm{n},
$$

implicaría realizar cálculos excesivamente laboriosos, y también a la necesidad de la búsqueda de un nuevo conocimiento que permita solucionar este problema. Ello es notorio a partir del tamaño de las magnitudes de los parámetros $\mathrm{n}=4000$ y $\mathrm{p}=0.001$, los cuales hacen improcedente el uso de dicha fórmula. Estos últimos aspectos determinan la naturaleza problemática de ambas situaciones.

Durante esta primera etapa pueden emplearse diferentes alternativas, según las condiciones de la enseñanza. Una variante puede apoyarse en el trabajo independiente, a partir de la orientación de la primera situación en una actividad anterior, donde los estudiantes tengan previamente información de las interrogantes a responder y cuando se enfrenten a la solución de la segunda situación descubran que las herramientas que disponen hasta el momento no son suficientes. Esta variante tiene la ventaja de racionalizar el uso del tiempo en el salón de clases.

La creación de situaciones problemáticas (etapa 2) contribuye a incentivar y potenciar el aprendizaje desarrollador, pues al mismo tiempo que se establece una contradicción entre lo conocido y la necesidad de desentrañar lo desconocido, se crea un motivo estimulante para la búsqueda del nuevo conocimiento. Con el propósito de crear situaciones problemáticas que estimulen la reflexión de los estudiantes, e implicarlos en la resolución del problema generado por la segunda situación, pueden formularse interrogantes como las siguientes:

- ¿Podrá resolverse este problema utilizando la distribución Binomial?

- ¿Se podrá emplear una tabla estadística de cálculo de probabilidades para resolverlo? ¿Por qué?

- ¿Se podrá utilizar la función de distribución? ¿Por qué? ¿Será racional aplicar este procedimiento?

- ¿Cuál es la variable de interés? Clasifíquela.

- ¿Cuáles son los valores de los parámetros de la distribución?

- ¿Qué características poseen los parámetros $n$ y $p$ de esta distribución, en comparación con los valores utilizados en situaciones anteriores donde $n$ tenía un valor menor y $p$ un valor mayor? ( $n$ es un número muy grande y $p$ es un número pequeño, próximo a cero). 
- ¿Será conveniente buscar otra distribución de probabilidad que permita dar respuesta al problema?

Preguntas similares a las anteriores tienen carácter heurístico y pueden estimular la comunicación en el aula y favorecer un diálogo con los estudiantes, donde se revele la necesidad de adquirir un nuevo conocimiento; a saber, una nueva distribución probabilística.

Durante la presentación del nuevo contenido (etapa 3) se puede comentar la necesidad de introducir una nueva distribución probabilística asociada a cierta variable aleatoria discreta, relacionada estrechamente con la distribución binomial y cuya función de probabilidad es:

$$
p(x)=\frac{\lambda^{x} e^{-\lambda}}{x !}, \text { con } \mathrm{x}=0,1,2, \ldots,
$$

donde $\lambda$ es el parámetro de la distribución y representa el número promedio de sucesos por unidad de tiempo (o también longitud, superficie, volumen) y e $=2.71828 \ldots$ En esta definición debe destacarse que la variable aleatoria $x$ representa el número de sucesos que se producen en un intervalo continuo $t$ o en una región específica y que puede tomar un conjunto infinito numerable de valores.

Para dar continuidad a la solución del problema puede hacerse reflexionar a los estudiantes, a manera de generalización, que en la distribución binomial cuando $p$ es muy pequeño (menor que 0,1) y $n$ es muy grande (mayor que 50) el valor esperado en la distribución de Poisson resulta un valor aproximado adecuado del valor esperado en la distribución binomial y, por tanto, puede ser sustituido por este $(\lambda=n p)$.

Finalmente, la solución del problema puede llevarse a término de forma sencilla, usando la tabla estadística para el cálculo de probabilidades de la distribución de Poisson:

$$
\lambda=n p=8000 \times 0.001=8 \text {; luego } P(x<7)=P(x \leq 6)=0.313 .
$$

Podría usarse, también, un proceso inductivo para que el propio estudiante sea el que arribe a la conclusión de la relación que se establece entre los valores esperados de ambas distribuciones, a partir de la disminución de los valores de $p$ y el incremento de $n$. Esto podría orientarse como una actividad investigativa mediante el uso de las tablas estadísticas de la distribución binomial y de Poisson, donde se comparen los valores de probabilidad de la variable aleatoria en cada una de las distribuciones para valores escogidos de los parámetros.

La presente metodología culmina con la identificación conceptual en ejemplos de aplicación (etapa 4). Aquí el estudiante debe tener la posibilidad de reconocer situaciones que se modelen a través de distribuciones de Poisson. No solo es importante tener presente que se trata de eventos discretos, sino también identificar el intervalo o región específica donde tienen lugar los eventos. En las situaciones prácticas que se seleccionen es importante que el 
estudiante reconozca tres aspectos esenciales: la denominación del evento o tipo de variable, el intervalo o región específica donde ocurren los sucesos, y la magnitud en que se expresa el intervalo (tiempo, longitud, área etc.).

\subsection{La distribución de Poisson a partir de las propiedades de un proceso de Poisson}

Otra manera de abordar la enseñanza de la distribución de Poisson resulta de verla como un proceso en el cual ocurren sucesos discretos en un intervalo continuo de tiempo, longitud etc., de forma tal que si se acorta suficientemente el intervalo, tengan lugar tres condicionantes esenciales. Primero, que la probabilidad de observar exactamente un suceso en el intervalo sea estable. Segundo, que la probabilidad de observar dos o más sucesos en el intervalo sea cero. Y tercero, que la ocurrencia de un suceso en cualquier intervalo sea estadísticamente independiente de la ocurrencia de un suceso en cualquier otro intervalo de igual magnitud. Con este propósito se establece la siguiente secuencia de etapas en el tratamiento didáctico del contenido: (1) Presentación de ejemplos para incentivar el aprendizaje del nuevo contenido, (2) Introducción del nuevo contenido, y (3) Planteamiento de problemas de aplicación.

Se parte de ejemplos que, potencialmente, incentiven el aprendizaje del nuevo contenido (etapa 1). A partir de situaciones reales, similares a las ilustradas anteriormente, puede llamarse la atención sobre ciertas características de los ejemplos presentados. Para ello puede resultar útil la formulación de interrogantes similares a las siguientes:

- ¿Cómo se denomina la variable de interés o evento que ocurre?

- ¿Cómo se clasifica dicha variable?

- ¿En qué intervalo o región específica ocurren los eventos?

- ¿Cuál es la magnitud en que se expresa el intervalo o región específica?

Esto puede justificar la necesidad de estudiar una nueva distribución probabilística, de variable aleatoria discreta, estrechamente relacionada con la distribución binomial y que juega un papel importante en la solución de problemas de ingeniería.

Siguiendo la secuencia de ideas desarrolladas a través de las respuestas a las interrogantes anteriores, se introduce el nuevo contenido (etapa 2). Aquí se debe destacar que cada una de esas situaciones constituye un caso particular de lo que se denomina proceso aleatorio o proceso de Poisson. Lo que caracteriza a tales procesos es su dependencia del tiempo, o sea, el hecho de que ciertos eventos suceden o no (por azar) a intervalos regulares de tiempo o en un intervalo continuo de tiempo (FREUND; MILLER; JONSON, 2006). 
Seguidamente, se puntualizan tres propiedades esenciales que caracterizan todo proceso de Poisson; ellas son:

- La probabilidad de ocurrencia de un suceso o evento durante un intervalo muy pequeño $\Delta t$ está dada por $\theta \Delta t$, donde $\theta$ representa el número promedio de sucesos por unidad de tiempo.

- La probabilidad de ocurrencia de más de un suceso durante cada uno de los pequeños intervalos $\Delta t$ es despreciable, es decir, puede considerarse como nula.

- La probabilidad de ocurrencia de un suceso durante uno de tales intervalos no depende de lo que sucedió antes.

Generalmente, los procesos que cumplen tales propiedades vienen asociados a eventos que ocurren durante un intervalo dado y pueden generar observaciones para una variable aleatoria discreta $X$. Esta es la denominada variable aleatoria de Poisson, cuya distribución de probabilidad correspondiente se llama distribución de Poisson. Así, de forma predominantemente inductiva, es posible arribar a una definición formal del concepto que queda expresado en la fórmula correspondiente.

En función de lograr una mejor comprensión de la distribución de Poisson, es conveniente complementar el análisis con dos consideraciones importantes. Por una parte, la probabilidad de ocurrencia de un suceso en el intervalo es proporcional a la longitud del intervalo, lo que equivale a plantear la relación $P=\theta \Delta t$, donde $\Delta t$ indica un intervalo muy pequeño y $\theta$ una constante que representa el promedio de sucesos que ocurren en el intervalo. Por otra parte, la probabilidad de que ocurra más de un suceso en el intervalo pequeño es despreciable, lo cual se puede expresar mediante la relación $P \approx 0$.

La comprensión de las propiedades del proceso de Poisson resulta compleja si la explicación del contenido sólo se realiza a partir de formulaciones generales, pues ello exige un nivel elevado de abstracción. Un ejemplo detallado puede servir para puntualizar aspectos típicos de los problemas afines. Por ejemplo:

En la pizarra telefónica de cierta empresa se reciben llamadas aleatorias durante un intervalo continuo de una hora. Cualquier llamada que se reciba en ese intervalo representa la ocurrencia de un evento discreto. Si se conoce que se reciben 180 llamadas como promedio y se divide el intervalo de una hora en 3600 intervalos consecutivos de un segundo, se tendrá $\lambda$ $=\theta t=180 / 3600=0.05$. Por tanto, de aquí se infieren las siguientes observaciones:

- La cantidad esperada o promedio de llamadas recibidas en cualquier intervalo de un segundo es igual a 0.05 , es decir, permanece constante. 
- La probabilidad de recibir más de una llamada en cualquier intervalo de un segundo es cero.

- Recibir determinada llamada en cualquier intervalo de un segundo no tiene efecto sobre recibir una llamada en cualquier otro intervalo de un segundo. O sea, es estadísticamente independiente.

Finalmente, se procede a ejemplificar las ideas expuestas en problemas de aplicación (etapa 3). Un problema ilustrativo puede ser el siguiente:

Los índices de llegadas de los aviones son factores importantes que se deben tomar en cuenta para la seguridad en los aeropuertos. Suponga que los aviones pequeños llegan a cierto aeropuerto, de acuerdo con un proceso de Poisson, con un índice de seis arribos por hora.

a) ¿Cuál es la probabilidad de que exactamente cuatro aeronaves pequeñas lleguen durante un periodo de una hora?

b) ¿Cuál es la probabilidad de que al menos cuatro lleguen durante un periodo de una hora?

c) Si en este momento acaba de arribar una aeronave pequeña, ¿cuál es la probabilidad de que la próxima demore en arribar no más de dos días?

Las preguntas están formuladas con un nivel de complejidad que crece gradualmente. Primero se hace alusión a un caso bien determinado, luego a un intervalo, seguidamente se exige que los estudiantes apliquen el conocimiento aprendido ante nuevas situaciones de aprendizaje. En efecto, el último inciso se ha formulado con el propósito de incentivar un nuevo conocimiento, asociado a la distribución exponencial. Esta última está muy relacionada con la distribución de Poisson y bien pudiera considerarse como un punto de partida para nuevos contenidos.

De forma resumida, ambas metodologías difieren esencialmente en sus presupuestos didácticos. En efecto, la primera toma como vía la creación de situaciones problemáticas, mientras que la segunda se apoya en las relaciones dialécticas de inducción-deducción en la construcción del nuevo conocimiento. En ambos casos es posible una ejemplificación variada y profunda, lo cual favorece la transferencia o adaptación de los métodos de solución hacia problemas nuevos (CATRAMBONE; HOLYOAK, 1990).

\section{Implementando ambas metodologías en carreras de ingeniería}

Con la finalidad de comparar ambas metodologías, se decide su implementación en carreras de ingeniería. Un aspecto esencial para establecer el paralelo consiste en determinar 
en qué medida tiene lugar un aprendizaje desarrollador. Tal cuestión tiene una elevada carga de subjetividad, por lo cual se decide precisar el sentido de este desarrollo, partiendo de la definición asumida (CASTELLANOS et al., 2001).

\subsection{Cualificando la variable de investigación}

Específicamente, se determina la apropiación activa y creadora del contenido en el sentido del incremento de la participación en clases, así como en la resolución de problemas de aplicación y en la búsqueda de nuevos problemas. Es decir, un conjunto de aspectos básicamente objetivos como descriptores de un primer indicador. Los restantes aspectos (autoperfeccionamiento, socialización, compromiso y responsabilidad social) se enfocan desde una óptica predominantemente subjetiva. El cuadro 1 ilustra el procedimiento seguido en la evaluación del aprendizaje desarrollador. La escala de calificación responde a las categorías evaluativas comunes de la Educación Superior en Cuba: 2 = Mal, 3 = Regular, $4=$ Bien, y 5 = Excelente.

\begin{tabular}{|l|l|l|}
\hline \multicolumn{1}{|c|}{ Indicador conceptual } & \multicolumn{1}{|c|}{ Descriptores } & \multicolumn{1}{c|}{ Escala } \\
\hline $\begin{array}{l}\text { Apropiación activa y } \\
\text { creadora del contenido }\end{array}$ & $\begin{array}{l}\text { Participación en clases, resolución de } \\
\text { problemas de aplicación ingenieril, planteo y } \\
\text { reformulación de problemas. }\end{array}$ & Cualitativa ordinal: 2-5 \\
\hline $\begin{array}{l}\text { Exteriorización consciente } \\
\text { del contenido }\end{array}$ & $\begin{array}{l}\text { Afán por perfeccionar los resultados, } \\
\text { disposición a socializar el conocimiento, } \\
\text { compromiso con la profesión futura, } \\
\text { responsabilidad en el uso del conocimiento. }\end{array}$ & Cualitativa ordinal: 2-5 \\
\hline
\end{tabular}

Cuadro 1 - Evaluación del aprendizaje de la distribución de Poisson en un sentido desarrollador

Para la cualificación integral del aprendizaje desarrollador se suman los valores de ambos indicadores y se siguen los siguientes criterios ordinales: 4-5 = 2 (Mal), 6-7 = 3 (Regular), 8-9 (Bien), y $10=5$ (Excelente). Aquí la dinámica cualidad-cantidad parte del principio de que ambos indicadores tienen igual jerarquía. Si se diera mayor peso al primero de ellos, entonces se estaría evaluando el aprendizaje propiamente dicho y no su perspectiva desarrolladora.

\subsection{Sujetos y contexto}

La implementación de ambas metodologías se lleva a cabo en las carreras de Ingeniería Industrial e Ingeniería Informática de la UHOLM. El estudio empírico se desarrolla en el primer semestre del curso escolar 2011-2012, con la selección al azar de dos salones de cada carrera. El subconjunto de estudiantes representa, de forma típica, a este estrato 
ingenieril, por tener aproximadamente la misma edad, similar procedencia de bachillerato, semejante motivación por la profesión, entre otros aspectos que dan crédito sobre la homogeneidad de las muestras. Igualmente, los profesores tienen aproximadamente la misma experiencia, formación matemática y didáctica, y siguen el mismo programa de estadística en sesión de la mañana.

\subsection{Tratamiento}

Se establecen dos grupos experimentales, cada uno de los cuales está compuesto por dos salones de cada carrera (EXP_1, $\mathrm{N}_{1}=47$; EXP_2, $\left.\mathrm{N}_{2}=50\right)$, para la implementación respectiva de cada metodología (MET_1 y MET_2). Particularmente, se sigue el desarrollo del tema común Distribuciones Teóricas de Probabilidad (12 horas/clase distribuidas en tres semanas lectivas), con énfasis en el aprendizaje de la distribución de Poisson, que es la distribución de variable aleatoria discreta estudiada después de la distribución Binomial.

Se suministra una prueba de entrada y otra de salida, cada una de las cuales incluye dos problemas que requieren la identificación de la distribución y la selección de un método de resolución. La primera prueba evalúa el aprendizaje desarrollador (AD) de la distribución Binomial, mientras que la segunda lo hace para la distribución de Poisson. Aunque la prueba de entrada no evalúa específicamente el aprendizaje desarrollador de la distribución de Poisson, sirve de referente comparativo en el marco de las distribuciones estadísticas de probabilidad. La tabla 1 sintetiza la estructura del presente estudio.

Tabla 1 - Diseño del estudio

\begin{tabular}{ccccc}
\hline Grupo & N & Pretest & Tratamiento & Postest \\
\hline EXP_1 & 47 & AD (Binomial) & MET_1 & AD (Poisson) \\
EXP_2 & 50 & AD (Binomial) & MET_2 & AD (Poisson) \\
\hline
\end{tabular}

\subsection{Resultados y discusión}

En el orden descriptivo, al comparar cada grupo respecto a sí mismo, ambos casos incrementan los valores medios de la variable AD. La mejoría es ligeramente mayor en el grupo EXP_2 (diferencia longitudinal: $.32>.27$ ), donde se implementa la segunda metodología. Un comportamiento similar se aprecia al comparar los grupos tras cada test (diferencia transversal: $.16>.11$ ). En el transcurso del estudio, la desviación típica tiende a disminuir en el primer grupo $(.895<$.907) y a aumentar en el segundo $(1.016>.995)$. Los resultados aparecen representados en la tabla 2. 
Tabla 2 - Resultados de la aplicación de los test

\begin{tabular}{ccrc}
\hline Grupo & \multicolumn{1}{c}{ Pretest } & \multicolumn{1}{c}{ Postest } & Diferencia de las medias \\
\hline EXP_1 & $2.79(.907)$ & $3.06(.895)$ & .27 \\
EXP_2 & $2.90(.995)$ & $3.22(1.016)$ & .32 \\
\hline Diferencia de las medias & \multicolumn{1}{c}{.11} & .16 & \\
\hline
\end{tabular}

Nota. Las desviaciones típicas aparecen entre paréntesis.

Las evidencias empíricas sugieren las siguientes hipótesis de forma aparente:

H1: El aprendizaje desarrollador mejora en cada grupo, independientemente de la metodología que se implemente.

H2: El aprendizaje desarrollador tiene mejores resultados tras la implantación de la segunda metodología.

Para la contrastación de la primera hipótesis se aplica el test de Wilcoxon para muestras apareadas, ya que la escala de la variable AD es no métrica (cualitativa ordinal) y los datos permiten comparar los resultados de cada sujeto respecto a sí mismo. El mencionado test se aplica en cada grupo, comparando respectivamente la prueba de entrada con la de salida. Los datos son procesados utilizando el paquete estadístico SPSS® (v. 15.0.1).

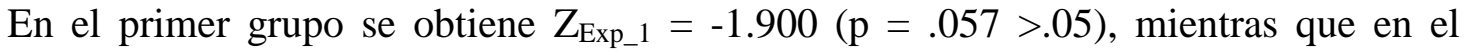
segundo grupo resulta $Z_{\text {Exp_2 }}=-2.717(\mathrm{p}=.007<.01)$. Por tanto, la hipótesis H1 solo se corrobora en el caso del segundo grupo. La aplicación de la prueba no paramétrica de los signos produce un resultado similar, con la particularidad de que se obtiene mejor nivel de significación en el segundo grupo.

En el caso de la segunda hipótesis, ya que antes y después de la intervención las muestras son independientes, se utiliza el test de Mann-Whitney para realizar la comparación. Para cada caso se obtienen valores similares del estadístico $U$ y fallan ambas significaciones asintóticas bilaterales $\left(\mathrm{U}_{\text {Pretest }}=1114.500, \mathrm{p}=.638>.05 ; \mathrm{U}_{\text {Postest }}=1080.500, \mathrm{p}=.475>.05\right)$. Por tanto, no es posible rechazar la hipótesis de nulidad relativa a H2.

Un análisis cualitativo de los resultados permite complementar el estudio. En este sentido, se destacan los siguientes aspectos:

- La participación de los estudiantes en las clases fue más elevada cuando se utilizó la Metodología 2; ello puede deberse a que esta vía permite introducir el nuevo contenido de forma más breve y sencilla y, a su vez, hacer un uso más racional del tiempo establecido, permitiendo que los estudiantes resuelvan un mayor número de problemas de aplicación. Sin embargo, la experiencia sugiere que el tratamiento de las propiedades de procesos de Poisson debe hacerse e ilustrarse a partir de los mismos problemas seleccionados, sin necesidad de acudir al uso de expresiones generales y abstractas que, frecuentemente, poco aportan al aprendizaje desarrollador. 
- En general, los estudiantes logran clasificar el tipo de variable y nombrarlas, así como las magnitudes de los intervalos usando cualquiera de ambas metodologías. Sin embargo, algunos presentan dificultades para identificar el tipo de problema, o sea, para decidir a partir del enunciado si se trata de un problema asociado a la distribución de Poisson o a la Binomial; sobre todo cuando el enunciado no explicita que la variable aleatoria sigue una distribución de Poisson. Independientemente de la naturaleza multilateral de este hecho, es notoria la necesidad que tienen los estudiantes de enfrentarse a un mayor número de problemas con variedad de enunciados. Ello concuerda con ciertos hallazgos de R. Catrambone y K. J. Holyoak, durante la enseñanza de problemas asociados a la distribución de Poisson. Tras dos experimentaciones, estos autores concluyeron que, en el contexto de la ejemplificación, es preferible discutir varias submetas necesarias para la solución de un problema nuevo, en lugar de varios métodos para establecer submetas en un problema nuevo (CATRAMBONE; HOLYOAK, 1990).

- Aunque en los alumnos de Ingeniería Informática se acentúa el grado de motivación por la estadística, en ambas carreras se aprecia que cuando los problemas están ligados a situaciones de interés profesional, la participación activa y el compromiso en la realización de las actividades es más elevado. La concepción de los grupos integrando salones de cada carrera, posibilitó un mayor acercamiento a la formación de ingenieros en sentido general.

\section{Conclusiones}

El presente estudio compara dos metodologías para la enseñanza de la distribución de Poisson en carreras de ingeniería. La primera de ellas toma como referente lógico-matemático la distribución de Poisson como aproximación a la distribución Binomial, mientras que la segunda sigue el enfoque de un proceso de Poisson. Partiendo de ambas posibilidades para el estudio de esta distribución de probabilidad, la primera metodología se basa en la problematización del contenido, mientras que la segunda se apoya en las relaciones dialécticas de inducción-deducción. 
Tras la implementación de los tratamientos experimentales se producen cambios favorables en el aprendizaje de las distribuciones, tanto en un grupo como en el otro; aunque solo en el segundo existen atisbos estadísticamente significativos de un aprendizaje desarrollador. Resulta atinado afirmar que lo determinante no reside en el hecho de que se problematice el contenido o se mueva el pensamiento en la dialéctica inducción-deducción. Lo determinante no está en la base ontológica del contenido, sino en el método que trasluce las potencialidades didácticas desde una perspectiva gnoseológica.

Ya que las evidencias empíricas no revelan superioridad de una metodología respecto a otra, estas pueden seleccionarse tomando en consideración el tiempo que se disponga, las motivaciones de los estudiantes, los métodos empleados en contenidos previos (pues siembre es plausible buscar cierto equilibrio), entre otros aspectos. A tono con lo anterior, la determinación de ambas metodologías adquiere por sí misma un valor práctico.

Con el objetivo de dar continuidad a la presente investigación, es conveniente diseñar nuevos estudios, de modo que se cualifique el aprendizaje de la distribución de Poisson en cada test (entrada y salida). En el caso descrito, se realizan comparaciones respecto al aprendizaje de la distribución Binomial, lo cual desvía el análisis hacia el estudio de las distribuciones en general, y no precisamente hacia la de Poisson en particular. No obstante, es positivo el hecho de que la comparación tome como referente el aprendizaje desarrollador de conceptos probabilísticos similares en el marco de la formación de ingenieros.

Otro aspecto importante consiste en la necesidad de perfeccionar la escala de medición. El uso de los cuatro niveles (2-5) se ajusta a una práctica viable instaurada en la educación superior cubana, pero tratándose de un estudio científico puede refinarse más hasta lograr mayor nivel de precisión en la cualificación. Incluso, los indicadores conceptuales también requieren de mayor atención, no solo por el enriquecimiento de los descriptores sino también por la objetividad en la integración de sus valores de escala.

Resulta llamativo el hecho de que la desviación típica tienda a disminuir en el primer grupo y a aumentar en el segundo. Una primera impresión sugiere que las diferencias individuales se acentúan más en un ambiente de inducción-deducción, pero menos en uno que enfatiza la problematización. Sin embargo, tal especulación enfrenta el obstáculo de la limitada estratificación de la escala, lo cual la hace hipersensible amén de que su naturaleza no métrica también impide un análisis de varianza.

\section{Referencias}


ACOSTA, F. M. Hints for the improvement of quality teaching in introductory engineering statistics courses. European Journal of Engineering Education, Brussels, v. 25, n. 3, p. 263-280, Jul. 2000.

BISGAARD, S. Teaching statistics to engineers. The American Statistician, Alexandria, v. 45, n. 4, p. 274-283, Nov. 1991.

BLANCO, A. Una revisión crítica de la investigación sobre las actitudes de los estudiantes universitarios hacia la Estadística. Revista Complutense de Educación, Madrid, v. 19, n. 2, p. 311-330, Aug. 2008.

BORASI, R. On the nature of problems. Educational studies in mathematics, Amsterdam, v.17, n. 2, p. 125-141, May 1986.

BORREGO, M.; DOUGLAS, E. P.; AMELINK, C. T. Quantitative, qualitative, and mixed research methods in engineering. Journal of Engineering Education, Washington, v. 98, n. 1, p. 53-66, Feb. 2009.

CASTELLANOS, D.; CASTELLANOS, B.; LLIVINA, M. J.; SILVERIO, M. Hacia una concepción del aprendizaje desarrollador. La Habana: Pueblo y Educación, 2001.

CATRAMBONE, R.; HOLYOAK, K. J. Learning subgoals and methods for solving probability problems, Memory \& Cognition, New York, v. 18, n. 6, p. 593-603, Jul. 1990.

BENDELL, A.; DISNEY, J.; McCOLLIN, C. The future role of statistics in quality engineering and management. The Statistician, Hoboken, v. 48, n. 3, p. 299-326, Sep. 1999.

DYCK, J. L.; GEE, N. A. A sweet way to teach students about the sampling distribution of the mean, Teaching of Psychology, Thousand Oaks, v. 25, n. 3, p. 192-195, Jul. 1998.

FREUND, J. E.; MILLER, I. R.; JOHNSON, R. Probabilidades y Estadística para Ingenieros. La Habana: Félix Varela, 2006.

HECKEL, R. W. Current and emerging statistical trends in engineering education. Journal of Engineering Education, Washington, v. 83, n. 4, p. 1-7, Oct. 1994.

HOGG, R. V. A core in statistics for engineering students. The American Statistician, Alexandria, v. 48, n. 4, p. 285-287, Nov. 1994.

JOHNSON, E.; ENGERER, B.; LEITCH, K.; TOUGAW, D. Teaching probability and statistics in a firstyear engineering course. In: FRONTIERS IN EDUCATION CONFERENCE, $38^{\text {th }}, 2008$, New York.

Proceedings... New York: Saratoga Springs, 2008. p. 22-25.

KVAM, P. H. The effect of active learning methods on student retention in engineering statistics. The American Statistician, Alexandria, v. 54, n. 2, p. 136-140, May 2000.

LIPSON, K. The role of the sampling distribution in understanding statistical inference. Mathematics Education Research Journal, Berlin, v. 15, n. 3, p. 270-287, Dic. 2003.

MAAT, S. M.; ZAKARIA, E. The learning environment, teacher's factor and students attitudes towards mathematics among engineering technology students. International Journal of Academic Research, Baku, v. 2, n. 2, p. 16-20, Mar. 2010.

MES (MINISTERIO DE EDUCACIÓN SUPERIOR). Reglamento. Trabajo Docente y Metodológico. Resolución Ministerial 210/2007, La Habana: MES, 2007.

OLIVO, E. Significado de los intervalos de confianza para los estudiantes de ingeniería en México. 2008. 340f. Tesis (Doctorado en Didáctica de la Matemática) - Universidad de Granada, Granada, 2008. Disponible en: http://digibug.ugr.es/bitstream/10481/2063/1/17675947.pdf. Consultado el: 07 feb. 2013. 
READING, C.; REID, J. An emerging hierarchy of reasoning about distribution: From a variation perspective. Statistics Education Research Journal, The Hague - Leidschenveen, v. 5, n. 2, p. 46-68, Nov. 2006. Disponible en: http://iase-web.org/documents/SERJ/SERJ5\%282\%29_Reading_Reid.pdf. Consultado el: 09 feb. 2013.

ROMERO, R.; FERRER, A.; CAPILLA, C.; ZUNICA, L.; BALASCH, S.; SERRA, V.; ALCOVER, R.Teaching Statistics to Engineers: An Innovative Pedagogical Experience. Journal of Statistics Education, Alexandria, v. 3, n. 1, Mar.1995. Disponible en:

http://www.amstat.org/publications/jse/v3n1/romero.html. Consultado el: 09 nov. 2012.

SALDANHA, L. A., \& THOMPSON, P. W. Exploring connections between sampling distributions and statistical inference: An analysis of students' engagement and thinking in the context of instruction involving repeated sampling. International Electronic Journal of Mathematics Education, Istambul, v. 2, n. 3, Oct. 2007.

SORGE, C.; SCHAU, C. Impact of engineering students' attitudes on achievement in statistics: A structural model. In: ANNUAL MEETING OF THE AMERICAN EDUCATIONAL RESEARCH ASSOCIATION, $21^{\text {st }}$, 2002, Nueva Orleans. Proceedings... New Orleans. p. 1-21. Disponible en: http://evaluationandstatistics.com/AERA2002.pdf. Consultado el: 09 nov. 2012.

WATSON, J. M. The influence of variation and expectation on the developing awareness of distribution. Statistics Education Research Journal, The Hague - Leidschenveen, v. 8, n. 1, p. 32-61, May 2009. Disponible en: http://iase-web.org/documents/SERJ/SERJ8(1)_Watson.pdf. Consultado el: 09 nov. 2012.

WILD, C. The concept of distribution. Statistics Education Research Journal, The Hague Leidschenveen, v. 5, n. 2, p. 10-26, Nov. 2005. Disponible en: http://iaseweb.org/documents/SERJ/SERJ5(2)_Wild.pdf. Consultado el: 09 nov. 2012.

ZEWOTIR, T. \& NORTH, D. Focus on the statistical education of prospective engineers in South Africa. Pythagoras, Pretoria, v. 65, p. 18-23, Jun. 2007. 